\title{
Exploring brain function from anatomical connectivity
}

\author{
Gorka Zamora-López ${ }^{1,2 *}$, Changsong Zhou ${ }^{3,4}$ and Jürgen Kurths $s^{1,2,5,6}$ \\ 1 Bernstein Center for Computational Neuroscience, Berlin, Germany \\ 2 Department of Physics, Humboldt University, Berlin, Germany \\ ${ }^{3}$ Department of Physics, Hong Kong Baptist University, Hong Kong, China \\ ${ }^{4}$ Center for Nonlinear Studies, Hong Kong Baptist University, Hong Kong, China \\ 5 Transdisciplinary Concepts and Methods, Potsdam Institute for Climate Impact Research, Potsdam, Germany \\ ${ }^{6}$ Institute for Complex Systems and Mathematical Biology, University of Aberdeen, Aberdeen, UK
}

Edited by:

Claus Hilgetag, Jacobs University

Bremen, Germany

Reviewed by:

Marcus Kaiser, Seoul National University, South Korea

Luciano da F. Costa, Institute of Physics

of São Carlos, Brazil

${ }^{*}$ Correspondence:

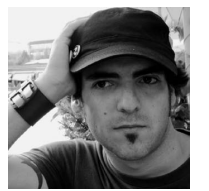

Gorka Zamora-López is a young scientist whose research interests lie at the cross-roads between neuroscience and the theory of complex networks. He is particularly interested in understanding behaviour from a global and integrative perspective. Dr. Zamora-López studied theoretical physics at the University of the Basque Country, Spain, and biophysics at the University of Oulu. After finishing his Ph.D. at the University of Potsdam, Germany, he currently works at the Bernstein Center for Computational

Neuroscience in Berlin.

gorka.zamora@ymail.com

The intrinsic relationship between the architecture of the brain and the range of sensory and behavioral phenomena it produces is a relevant question in neuroscience. Here, we review recent knowledge gained on the architecture of the anatomical connectivity by means of complex network analysis. It has been found that cortico-cortical networks display a few prominent characteristics: (i) modular organization, (ii) abundant alternative processing paths, and (iii) the presence of highly connected hubs. Additionally, we present a novel classification of cortical areas of the cat according to the role they play in multisensory connectivity. All these properties represent an ideal anatomical substrate supporting rich dynamical behaviors, facilitating the capacity of the brain to process sensory information of different modalities segregated and to integrate them toward a comprehensive perception of the real world. The results here exposed are mainly based on anatomical data of cats' brain, but further observations suggest that, from worms to humans, the nervous system of all animals might share these fundamental principles of organization.

Keywords: hierarchical networks, cortical networks, brain connectivity, integration, segregation, connectome, cortical hubs

\section{INTRODUCTION}

Since the discovery of neurons by the Spanish histologist Santiago Ramón y Cajal at the end of the nineteenth century, we have gained tremendous understanding on the composition and function of the nervous system: from the working of individual neurons to the principles of sensory perception. As the physical substrate at which all neural information processes happen, it is relevant to understand how the architecture of the brain manifests and supports the diversity of behaviors it generates: reflexes, emotions, perception, memory storage and retrieval, cognitive processes, etc. Unfortunately, comprehensive data of anatomical connectivity is still scarce and difficult to obtain. Achieving a detailed map of all the neurons and their interconnections in a mammalian brain is simply out of technological reach. Motivated by the available experimental techniques, for the most part of the twentieth century a notion of cortical function has prevailed in which different parts specialize in performing particular processing of the sensory input. This perspective has been well founded by physiological, surgical, and lesion studies (Kandel et al., 2000; Bear et al., 2006).

During the last decades, the advent of new data have questioned the limitations of that notion and have uncovered richer and more complex mechanisms of the working brain. A prominent problem is that a collection of specialized functions alone cannot give rise to a coherent perception of the reality. For that, different 
Segregation

It refers to the subdivision of the brain into regions specialized in particular functional tasks. This allows the brain to process information in parallel, simultaneously by distinct populations of neurons. Structurally, segregation implies that brain regions specialized in a particular function can be localized, and that this localization is rather stable across subjects and species.

\section{Integration}

Integration is the capacity of a system to collect information of different nature and combine it to produce new, useful, information. Sensory perception requires the binding of the features of a receptive field, e.g., color, orientation, and position of a visual object. A pre-requisite for awareness is that the brain integrates the information from the distinct sensory systems.

\section{Complex network}

Empirical networked systems are coined with the term complex because the connections between their components are neither randomly nor regularly organized. They have some degree of internal, non-trivial organization that is only revealed after extensive analysis.

\section{Adjacency matrix}

A network composed of $N$ nodes and $L$ links can be encoded into an $N \times N$ matrix whose elements $A_{i j}=1$ if there is a link connecting nodes $i$ and $j$, and $A_{i j}=0$ otherwise. The characterization of the network is mathematically performed by statistical description of its adjacency matrix.

\section{Scale-free distribution}

Also known as "power law," refers to a statistical distribution of the form $p(x) \sim x \gamma$. It presents scale invariances, i.e., multiplying the variable by a constant the form of the distribution does not change: $p(c x) \propto p(x)$. When plotted in a log-log plot scale-free distributions follow a descending line whose slope is the exponent $\gamma$.

\section{Clustering coefficient}

It is a network measure which characterizes the probability that the neighbors of one node, are also connected with each other. It is easily understood in social terms: two persons are more likely to know each other when they have a common friend or colleague. parts of the brain need to communicate and their information needs to be combined (Damasio, 1989; Tononi, 2004). Physiological recordings with multiple electrodes have revealed that distant neurons can synchronize (Fahle, 1993; Singer and Gray, 1995; Bressler and Kelso, 2001; Engel and Singer, 2001; Uhlhaas et al., 2010), and neuroimaging studies have extensively reported the co-activation of distant brain regions under different experimental conditions (Tass et al., 1998; Varela et al., 2001; Achard et al., 2006). These observations have set the foundations for novel approaches to understand the brain: that networks of segregated but interacting processes govern neural dynamics on top of the processing of the specialized regions. At the hidden ground of those functional and dynamic observations lies the fact that the neurons in a nervous system form a vast network with a mixture of both local and long-range connections.

In the present review, we describe the discoveries on the complex architecture of anatomical neural and cortical networks, and we discuss their impact on brain function. We concentrate on the data of large-scale connectivity between cortical areas in cats and macaque monkeys. We outline human connectivity data and the neural network of the worm Caenorhabditis elegans. In particular we discuss how the main structural properties of these networks facilitate the segregation/integration problem that the brain faces, i.e., its capacity to process sensory information in parallel and to combine it toward a coherent representation of the external world. In Section 2 we review basic concepts of complex networks. We also introduce a novel parametrization to characterize the roles of nodes in modular networks. In Section 3 essential properties of characteristic anatomical connectivity datasets are introduced. Section 4 summarizes the architectural features of the networks which support the problem of segregation and integration. In Section 5 novel results are presented which aim at classifying the cortical areas according to the multisensory nature of their connectivity.

\section{COMPLEX NETWORKS}

The recent finding of intricate patterns of interaction between the elements of many natural and artificial systems has altered the manner we understand complexity. The elements of a system can be physically interconnected, e.g., axonal projection between neurons or the cables between computer servers, which form the internet. Often, the connections represent abstract interactions, e.g., the friendship relations within a group of humans, the semantic association between two words, or the dynamical correlation between two brain regions.

A network representation provides the system with a form (a topology) which is mathematically tractable, Figures 1A-C, allowing to describe the system's architecture and to interpret its functional implications. The elements of the system are referred as nodes and the connections as links. This information is encoded into the adjacency matrix of the network. Several statistical descriptors exist to describe different scales of organization, from local properties of individual nodes to the large-scale architecture of the system (Newman, 2003; Boccaletti et al., 2006; Junker and Schreiber, 2008). The degree $k(i)$ of node $i$ is the number of nodes it is connected with. The distribution of the degrees $p(k)$ informs about the heterogeneity of the nodes. In a regular lattice all nodes have the same number of neighbors, hence they are statistically indistinguishable. On the contrary, real networks usually display broad degree distributions, near to scale-free; most of the nodes make a small number of connections but few nodes are highly connected. The latter nodes are referred as hubs. Other descriptors to characterize the importance of individual nodes include the betweenness centrality, which quantifies the centrality of a node for the flow of information within a network.

The distance $d_{i j}$ between two nodes $i$ and $j$ is defined as the number of links crossed when traveling from node $i$ to node $j$; see Figure 1B. The average path-length $l$ is the average of all pair-wise distances, $l=\left\langle d_{i j}\right\rangle$. The term small-world networks arises from the observation during the 1960s that lof a real social network was very short compared to its size, $l \sim \ln N=N$ where $N$ is the number of nodes (Travers and Milgram, 1969; Wasserman and Faust, 1994). Later on, in their seminal paper Watts and Strogatz (1998) reported another common property of real networks, a large clustering coefficient. It is now common in the literature to refer as small-world networks to networks with both short path-length and large clustering coefficient.

However, a large clustering is usually the trivial statistical consequence of another, more relevant feature found in most real networks: their modular structure. A module or community is defined as a subset of nodes which are densely interconnected to each other, but are sparsely connected to the nodes in other modules. Similarly, the modules may join to form larger modules and give rise to hierarchies. See Figure 1D for illustration. In real networks, and particularly in biological systems, network modules naturally reveal functional subdivision of the system. 


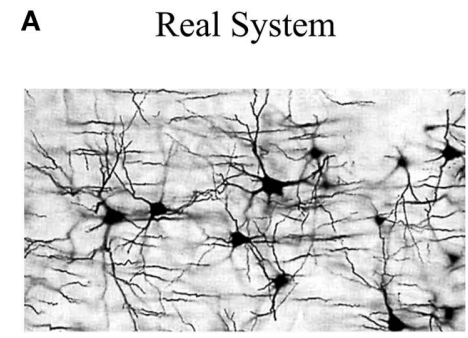

- $N=$ number of nodes

- $L=$ number of links

- Density, $\rho=\frac{2 L}{N(N-1)}$
B

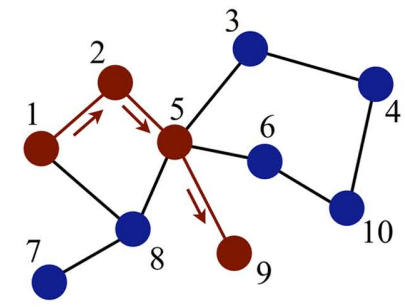

- Degree of node 10: $k_{10}=2$

- Distance between 1 and 9: $d_{1,9}=3$
C Adjacency Matrix, $\boldsymbol{A}_{i j}$

\begin{tabular}{|l|l|l|l|l|l|l|l|l|l|l|}
\hline & $\mathbf{1}$ & $\mathbf{2}$ & $\mathbf{3}$ & $\mathbf{4}$ & $\mathbf{5}$ & $\mathbf{6}$ & $\mathbf{7}$ & $\mathbf{8}$ & $\mathbf{9}$ & $\mathbf{1 0}$ \\
\hline $\mathbf{1}$ & 0 & $\mathbf{1}$ & 0 & 0 & 0 & 0 & 0 & $\mathbf{1}$ & 0 & 0 \\
\hline $\mathbf{2}$ & $\mathbf{1}$ & 0 & 0 & 0 & $\mathbf{1}$ & 0 & 0 & 0 & 0 & 0 \\
\hline $\mathbf{3}$ & 0 & 0 & 0 & $\mathbf{1}$ & $\mathbf{1}$ & 0 & 0 & 0 & 0 & 0 \\
\hline 4 & 0 & 0 & $\mathbf{1}$ & 0 & 0 & $\mathbf{1}$ & 0 & 0 & 0 & 0 \\
\hline $\mathbf{5}$ & 0 & $\mathbf{1}$ & $\mathbf{1}$ & 0 & 0 & $\mathbf{1}$ & 0 & $\mathbf{1}$ & $\mathbf{1}$ & 0 \\
\hline $\mathbf{6}$ & 0 & 0 & 0 & $\mathbf{1}$ & $\mathbf{1}$ & 0 & 0 & 0 & 0 & $\mathbf{1}$ \\
\hline $\mathbf{7}$ & 0 & 0 & 0 & 0 & 0 & 0 & 0 & $\mathbf{1}$ & 0 & 0 \\
\hline $\mathbf{8}$ & $\mathbf{1}$ & 0 & 0 & 0 & $\mathbf{1}$ & 0 & 0 & 0 & 0 & 0 \\
\hline $\mathbf{9}$ & 0 & 0 & 0 & 0 & $\mathbf{1}$ & 0 & 0 & 0 & 0 & 0 \\
\hline 10 & 0 & 0 & 0 & 0 & 0 & $\mathbf{1}$ & 0 & 0 & 0 & 0 \\
\hline
\end{tabular}

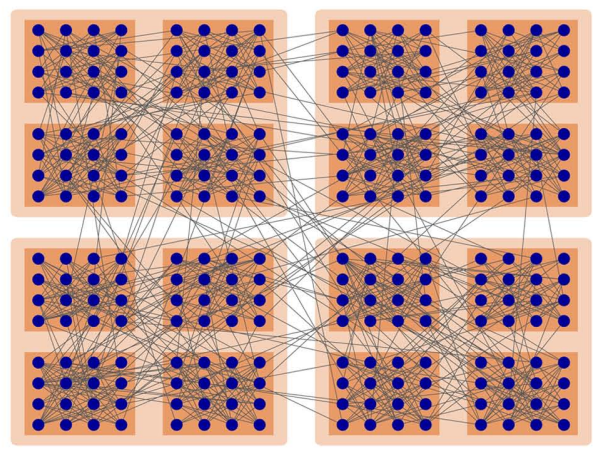

E

Roles of nodes

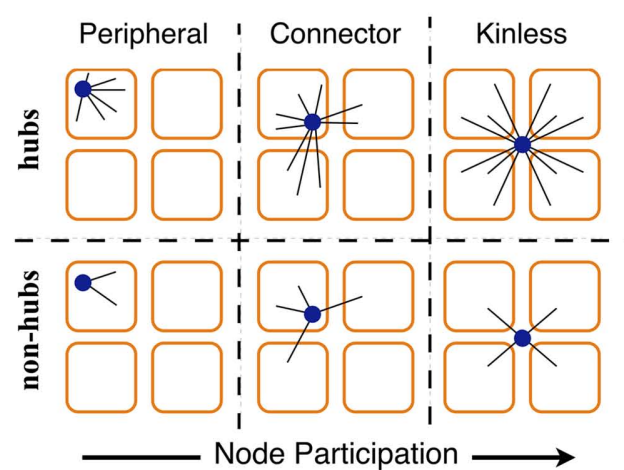

FIGURE 1 | Complex networks. (A-C) Some real systems can be abstractly represented by networks. The components of the system are replaced by nodes and their channels of interaction by links. Networks are mathematically encoded into adjacency matrices for their statistical analysis. (D) The nodes of a network often form densely interconnected modules, which can group together to form larger modules and hierarchies. (E) Given a network with modular organization its nodes can take different positions. These can be characterized by two parameters: (i) the global importance of the node or hubness, and (ii) the dispersion of the links of the node throughout the modules, referred as node's participation.

\section{ROLES OF NODES IN MODULAR NETWORKS}

Because of the modular organization, nodes can take different topological roles as is sketched in Figure 1E. Guimerà and Amaral (2005) proposed a framework consisting of two parameters to map the role of each node. One parameter, the withinmodule-degree, evaluates the internal importance of the node within its own module. The second parameter, the participation index $P_{i}$, evaluates how its links are distributed across modules. $P_{i}=0$ if all the links of $i$ are devoted to only one module (perfectly peripheral), and $P_{i} \rightarrow 1$ when its links are uniformly distributed throughout modules (perfectly kinless).

Motivated by this framework, we introduce an alternative set of parameters which overcome limitations of the original ones and provide additional information. First, we characterize the importance (hubness) of a node by its degree $k(i)$ rather than by its internal degree, which is restricted to the links within only one module. In order to compare systems of different sizes, we normalize the degree by the number of nodes in the network, $\langle K(i)\rangle=k(i) / N$.

An adequate normalization of the participation index is more elaborated. Given a network with $n$ modules, we assign an $n$-dimensional participation vector $\boldsymbol{P}_{i}$ to every node. The elements $P_{i, \text { }}$ represent the probability that the node $i$ belongs to the module $s$, where $s=1,2, \ldots, n$. This probability is expressed as $P_{i, s}=k_{i, s} / N_{s}$, where $k_{i, s}$ is the number of links which node $i$ makes to nodes in the community $s$, and $N_{s}$ is the size of the module $s$. In the schematic diagram of Figure $1 \mathrm{E}$ with four modules, a perfectly peripheral node would be assigned the participation vector $\boldsymbol{P}_{i}=(1,0,0,0)$, $\boldsymbol{P}_{i}=(0,1,0,0)$, etc. A perfectly kinless node would be assigned the participation vector $\boldsymbol{P}_{i}=(1 / 4,1 / 4$, $1 / 4,1 / 4)$.

Participation vectors contain all the statistical information about the role of the nodes according to the modular organization of the network. But for visualization, we need to reduce their dimensionality. Algebraic transformations such as the 
"truncated singular value decomposition" provide an optimal reduction (Arenas et al., 2010). Here we aim at reducing the information into a single scalar. Therefore we use the standard deviation $\sigma\left(P_{i}\right)$ of the elements in the vector and define the participation index as:

$P_{i}^{\prime}=1-\frac{n}{\sqrt{n-1}} \sigma\left(\boldsymbol{P}_{i}\right)$,

Our definition accounts for the fact that nodes are more likely to connect to larger modules than to smaller modules. The normalization guarantees that $P_{i}^{\prime}=0$ if and only if all the $k(i)$ links of node $i$ are devoted to one module, and $P_{i}^{\prime}=1$ if and only if its links are equally likely distributed over all the $n$ modules.

\section{DATASETS OF ANATOMICAL CONNECTIVITY}

Extracting a complete map of every neuron and their axonal projections in a mammalian brain is currently out of technological reach. Nevertheless, qualitative and statistical information on the cytoarchitecture of different parts of the brain reveal important insights about their function. Neurons in the cerebral cortex form distinguishable layers. This layered organization is not homogeneous; differences in the density of neurons and in the characteristics of the laminar structure are found. The laminar structure is more prominent in regions of early sensory processing such as primary sensory areas while areas of the prefrontal cortex lack of a clear layered organization (Fuster, 2003; Hilgetag and Grant, 2010).

The most accurate dataset of neuronal connectivity available is that of the worm C. elegans. Its nervous system has been fully mapped by reconstruction of electron micrographs of sectioned specimens (White et al., 1986; Durbin, 1987; Varshney et al., 2011). The network contains approximately 300 neurons and 3000 connections, including electrical junctions and chemical synapses between neurons, and neuromuscular junctions (www.wormatlas.org).

At larger scales, axonal fibers between regions of the brain can be discovered by tract-tracing experiments. A chemical tracer is injected into a small brain region and it is then transported along the cell's axon by intracellular transport mechanisms. After the animal is sacrificed, its brain is sectioned and stained what permits to track the propagation sites of the injected tracer. During the early 1990s application of such methods gave rise to a comprehensive description of the connectivity between cortical areas in the brains of cats (Scannell and Young, 1993), macaque monkeys (Young, 1993), and rats (Burns and Young, 2000). These networks are composed by a parcelation of the cerebral cortex of one hemisphere into cortical areas and the long-range fiber projections between them. Interhemispheric connections are discarded. The network of the cat is formed by approximately $N=53$ areas and $L=826$ directed projections, although its usage slightly varies across publications, some works even include the cortico-thalamic projections (Scannell et al., 1999). The network of the macaque comprises of up to $N=70$ cortical areas and $L=750$ links (Young, 1993), of them approximately 30 areas and 300 links (Young, 1992), near half of the network, correspond to visual areas. Updates of macaque's cortical connectivity are given in www. cocomac.org (Stephan et al., 2001). For rats'brain, only a partial connectivity dataset is available containing $N=23$ structures which are believed to be relevant for spatial navigation (Burns and Young, 2000).

The invasive and toxic nature of tract-tracing techniques make them unsuitable for application in human subjects. Large-scale connectivity of the human brain can be obtained by non-invasive diffusion-imaging techniques. The reliability of these methods to achieve accurate maps of human tractography is limited due to technical issues, e.g., the difficulty to distinguish between crossing and closely running parallel fibers (Basser and Jones, 2002; Dyrby et al., 2007; Gigandet et al., 2008; Chung et al., 2011). Nonetheless, they comprise the only techniques for tracing white matter pathways in a living brain and they serve to obtain an initial "draft" of the human large-scale connectivity (Sporns et al., 2005; Hagmann et al., 2008).

Analysis of these networks has led to the discovery of striking characteristics of functional relevance. First, these networks are densely connected. Although far from an all-to-all connected system, approximately $30 \%$ of all possible links are present. As a consequence, cortical areas are all at very few processing steps of each other. In the cortex of the cat, $30 \%$ of the pairs of areas are connected by direct links, and $60 \%$ are separated by only two processing steps (Zamora-López et al., 2009). Second, the path of information transmission between two cortical areas is not unique, but there exists many alternative routes through which information can flow (ZamoraLópez et al., 2009).

These two observations support the notion that the cerebral cortex is a highly interactive information processing system, and is dynamically flexible. Although cortical regions might specialize in the processing of a particular function, they do not operate independently, but in strong influence of each other. The fact that information can flow through different alternative paths 
significantly enhances the richness and complexity of the processing capabilities of the system with a limited number of resources. If the system were provided with mechanisms to selectively activate or inactivate paths of communication, the range of dynamical states it can host increases significantly. In the brain, inhibitory connections may very well be responsible for such switching dynamics and permit that the resources used by each process self-organize by mutual and transitory competition.

\section{SEGREGATION AND INTEGRATION OF SENSORY INFORMATION}

Sensory neurons encode physicochemical information into electrical signals which propagate through the nervous system undergoing several processing stages. As they are specialized in the detection and transduction of only one type of modal information (visual, auditory, proprioception, etc.), information about the different modalities remains separated along the paths of processing. At the cortex, these separated paths are projected into distinct regions where neuronal groups detect features of the sensory stimuli, e.g., color and orientation of visual objects, the frequency of auditive signals or the location at the body surface of a somatosensory stimulation. However, in order to generate a coherent perception of the reality, the brain needs to combine (integrate) this multisensory information at some place (Robertson, 2003) and during some time (Fahle, 1993; Singer and Gray, 1995; Engel and Singer, 2001). For this to happen, the paths of information need to converge.

Whether integration occurs in specialized and localized regions of the brain, resembling information processing of sensory features by specialized regions, or it happens as a consequence of distributed but coordinated processing in multiple areas is still a subject of debate. During the last decades, multi-electrode recordings have demonstrated that distant regions of the brain undergo transient states of correlated activity as the consequence of behavioral responses to sensory stimuli and cognitive tasks in non-human primates (Goldman-Rakic, 1988; Bressler, 1995; Fuster, 2003). Current neuroimaging techniques permit to observe the whole brain at work, revealing the occurrence of patterns of correlated activity between distributed cortical areas (Varela et al., 2001; Bullmore and Sporns, 2009; Bressler and Menon, 2010).

From an anatomical point of view, it has been argued that the functional capacity of the nervous system to balance between segregation (specialization) and integration is facilitated by its structural organization (Sporns and Tononi, 2001; Sporns et al., 2004; Kaiser, 2007; Shanahan, 2007). In the following, we review recent observations supporting this idea.

\subsection{SPECIALIZATION LEAD TO SEGREGATION}

Network clustering algorithms detect groups of nodes, or modules, which are densely interconnected and sparsely connected to nodes in other groups. As a result the algorithms return a partition of the nodes into distinct modules; every node is assigned to only one module. The areas in the cortico-cortical networks of the cat and macaque form a few distinguishable network modules, as well as the neurons in the neural network of the worm C. elegans. Although the algorithms make use only of connectivity information, the resulting modules are composed of cortical areas or neurons which are functionally related.

In the case of the cat connectivity, the network is arranged into four modules containing areas predominantly specialized in the processing of either visual, auditory, or somatosensory-motor information, and another module composed of frontolimbic areas (Scannell and Young, 1993; Hilgetag et al., 2000; Hilgetag and Kaiser, 2004). The difference in inter- and intra-connection densities elucidates how the networked architecture of the cerebral cortex helps keeping sensory information of different modalities segregated from each other, allowing for their parallel and simultaneous processing. As schematically illustrated in Figure 2A, information of different sensory modalities enter the cortex at distinct regions, which in cooperation with its neighboring areas, process the modally relevant information of the stimulus. In this case "neighboring areas" refers both to the network neighbors in the same module, and to the neighboring areas at the cortical surface, Figure $2 \mathbf{B}$.

\subsection{CORTICAL HUBS LEAD TO INTEGRATION}

The possibility to distinguish cortical regions and modules of regions involved in particular functions opens the question of where and how does the brain performs integration. Further analysis of cortico-cortical connectivity has revealed architectural properties which help to answer the question.

Both networks of cat and of macaque contain highly connected areas, which are referred as cortical hubs (Zemanová et al., 2006; Zhou et al., 2006; Sporns et al., 2007; Hagmann et al., 2008; Zamora-López et al., 2009). Due to their small sizes and large link densities, cortical networks 


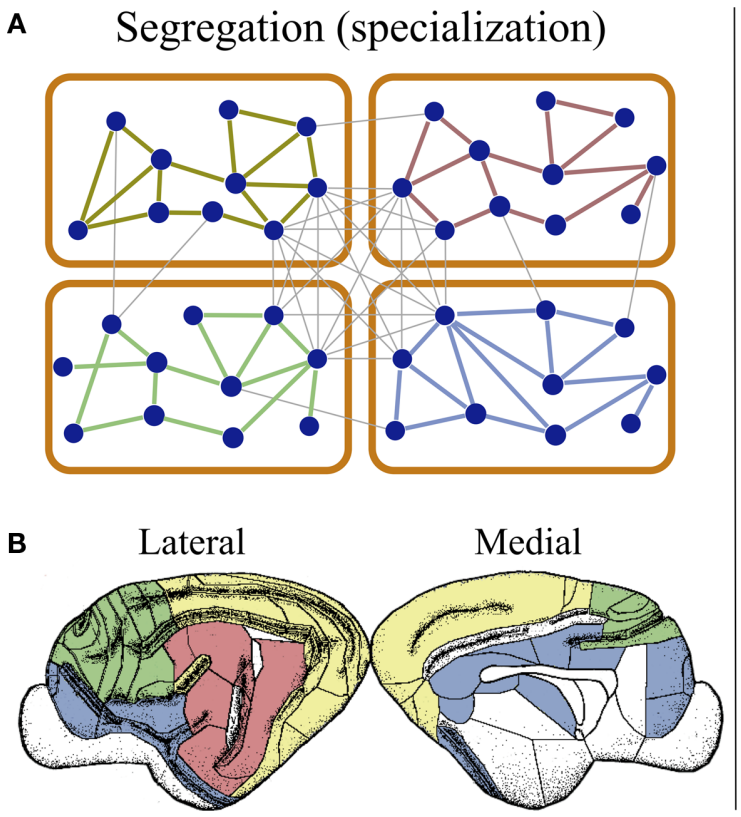

FIGURE 2 | Segregation and integration of multisensory information. (A) Cortico-cortical networks are organized into modules composed of areas devoted to the processing of information of one modality. This modular organization permits the brain to handle information of different modalities in parallel, at the same time by different regions. (B) At the cortical surface modaly related areas are found close to each other, as illustrated by the distribution of
C Integration
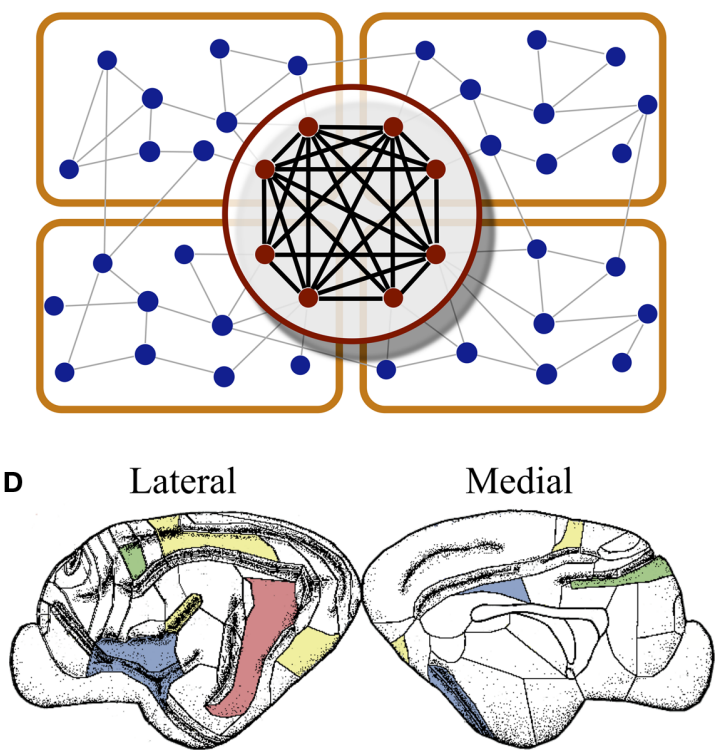

visual (yellow), auditory (red), somatosensory-motor (green), and frontolimbic (blue) areas in the cortex of cats. (C) Cortical hubs form a central module at the top of the cortical hierarchy, which is capable of integrating multisensory information as the coordinated activity of the hubs. (D) This module can only be detected by connectivity analysis because cortical hubs are dispersed throughout the cortical surface.
Rich-club

The rich-club is a network property that happens when the hubs of a network, the nodes with largest number of neighbors, are densely interconnected. Because of their large number of connections, hubs are likely to be connected, hence, a proper characterization of the rich-club requires to compare the result with a random probability of connections between the hubs. shall not be classified as scale-free, though they manifest properties of scale-free networks: a broad degree distribution and a similar robustness behavior after damage by removing nodes (Kaiser et al., 2007). Cortical hubs connect up to $60 \%$ of the areas, while hubs of typical scale-free networks (Barabási and Albert, 1999) link to no more than $0.1 \%$ of the nodes.

Cortical hubs play a crucial role in the multisensory communication by centralizing the paths of information between the modalities (ZamoraLópez et al., 2009). Beyond serving as bridges between modalities, cortical hubs have extensive afferent and efferent connections to areas in all the modules (visual, auditory, somatosensorymotor, and frontolimbic), what makes them suitable candidates as integrators. The question is then, whether cortical hubs operate independently, each specialized in integrating particular features, or they process multisensory information in cooperation.

In the cat's network, the cortical hubs are densely interconnected forming a structure known as a rich-club (Zamora-López et al., 2010). That is, the highly connected nodes are grouped into a module that occupies a central hierarchical role on top of the modular architecture; see Figure 2C. This observation strongly supports the idea that the information of different modalities is collected by the cortical hubs and combined in a cooperative manner.

To corroborate this idea, in Zamora-López et al. (2010) the authors showed a series of numerical experiments. Considering the network dynamics as a simple linear system at steady state, driven by Gaussian noise, and extending the concept of integration by Tononi et al. (1994), they measured: (i) the capacity of groups of areas to integrate information after simulated external sensory input, and (ii) the segregation suffered by the multimodal network after selective lesion of groups of areas. The results determined that the set of cortical hubs formed the optimal group of nodes in both cases; their collective behavior optimally integrate information in the presence of stimulation, and their simultaneous removal maximizes the damage to the network dynamics.

Finally, we shall emphasize a particularity of the cortical hubs. In contrast with common rules of neural organization, that functionally related areas fall into close regions of the cortical surface, Figure 2B, the cortical hubs form a functional module of the network which is dispersed throughout the cortex, as illustrated for the case of the cat in Figure 2D. 


\section{MULTISENSORY CHARACTERISTICS OF CORTICAL AREAS}

The nature of cortical areas containing cells responsive to multimodal stimulation has gained significant attention recently (Robertson, 2003; Wallace, 2004; Driver and Noesselt, 2008). Beyond multisensory integration, the effect of cross-modal connections can be associated to the sub-threshold modulation (inhibition or facilitation) of the neuronal activity in other modalities (Driver and Spence, 2000; Meredith et al., 2006; Allman et al., 2008). These cross-modal interferences may be regarded as the mechanism by which competition between modalities for an attentional focus is regulated.

In the following we investigate in more detail the multisensory nature of cortical areas, as it is reflected by their connectivity. We concentrate on the cortico-cortical network of the cat, which is composed of 52 cortical areas and the Hippocampus (Hipp), connected by 826 directed projections between them. The Hippocampus is the only subcortical brain region in this dataset and it is the least connected node, making only four efferent connections and receiving two afferent ones.
For a first illustration we show in Figure 3A the external degree of the cortical areas, that is, the number of links every area makes to areas in other modules. Equivalently, the internal degree is the number of links an area devotes to the areas in its module. The values displayed correspond to the averages of efferent and afferent connections. We find that most cortical areas participate in cross-modal communication with the exception of the primary visual cortex, area 17 , and the Hippocampus, which only connect to areas in their own corresponding module.

We now study the role of cortical areas within the modular and hierarchical structure of the network; see Figure 1E. Therefore, we map the areas into a space defined by two parameters; see Section 2 and Zamora-López (2009) for a detailed description. One parameter characterizes the importance of the node in the network. We choose the degree normalized by the number of nodes: $\langle K(i)\rangle=k_{i} / N$. The second parameter, the participation index $P_{i}^{\prime}$ of Eq. 1, characterizes how a node distributes its links throughout all modules in the network. It also accounts for the fact that the probability to connect to a module depends on the size of
A

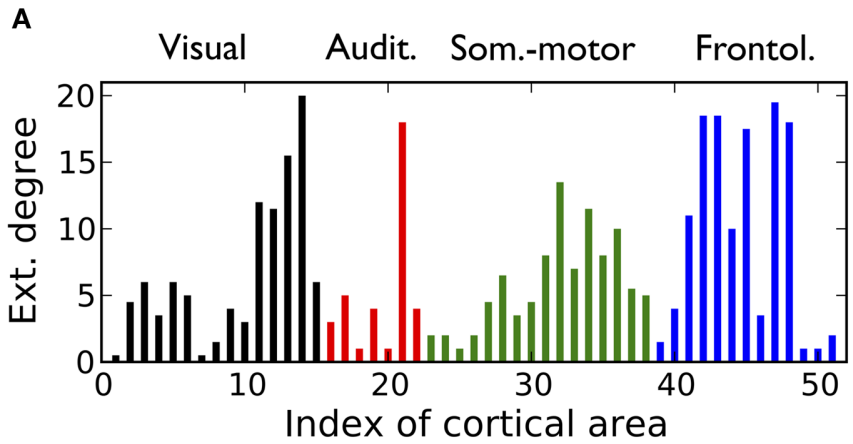

C

\begin{tabular}{|c|c|c|c|c|}
\hline & Visual & Auditory & Somato-Motor & Frontolimbic \\
\hline $\begin{array}{c}\text { Supramodal } \\
\text { hubs }\end{array}$ & $7,20 \mathrm{a}, 20 \mathrm{~b}, \mathrm{AES}$ & $\mathrm{EPp}$ & $6 \mathrm{~m}$ & $35,36, \mathrm{Ia}, \mathrm{Ig}, \mathrm{CGp}, \mathrm{PFCL}$ \\
\hline $\begin{array}{c}\text { Multimodal } \\
\text { areas }\end{array}$ & $\begin{array}{c}19^{*}, 20 \mathrm{~b}^{*}, 21 \mathrm{a}^{*}, 21 \mathrm{~b}, \\
\mathrm{ALLS}, \mathrm{AMLS}, \mathrm{DLS}, \\
\mathrm{PLLS}, \mathrm{PMLS}, \mathrm{PS}\end{array}$ & AII, Tem & $\begin{array}{c}\text { 5B1, 5Bm, SII, SIV }, \\
\text { SSSAi, SSAo }\end{array}$ & $\mathrm{CGa}, \mathrm{PFCMd}, \mathrm{RS}$ \\
\hline $\begin{array}{c}\text { Unimodal } \\
\text { areas }\end{array}$ & $17^{*}, 18^{*}, \mathrm{VLS}$ & $\begin{array}{c}\mathrm{AI}^{*}, \mathrm{AAF}^{*}, \\
\mathrm{P}^{*} \mathrm{VP}^{*}\end{array}$ & $1^{*}, 2^{*}, 3 \mathrm{a}, 3 \mathrm{~b}^{*}, 4 \mathrm{~g}$ & $\begin{array}{c}\text { Enr, Hipp, PFCMil, } \\
\mathrm{pSb}, \mathrm{Sb}\end{array}$ \\
\hline
\end{tabular}

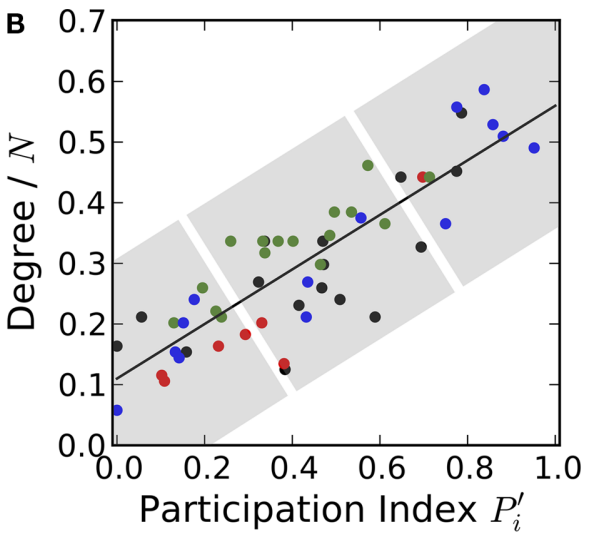

$\mathrm{pSb}, \mathrm{Sb}$
FIGURE 3 | Multisensory nature of cortical areas in cats' brain. (A) Number of connections that every cortical area makes to areas in other modalities. (B) The topological roles of the nodes display an ascending linear trend which is characteristic of the modular organization with centralized hierarchy of the network. This trend permits a classification of cortical areas into three categories (highlighted be shaded regions): unimodal areas, multimodal areas, and supramodal hubs (C). Areas marked with an asterisk ( $\left.{ }^{*}\right)$ contain either retinotopic, tonotopic, or somatotopic maps of the sensory input. 
the module. The parameter is normalized such that $P_{i}^{\prime}=0$ if the links of node $i$ are restricted to only one module (the node is peripheral, Figure $1 \mathrm{E}$ ), and $P_{i}^{\prime}=1$ only if its $k_{i}$ links are equally likely distributed over all modules (the node is kinless).

Applying these measures to the cortico-cortical network of the cat, Figure 3B, we find that the mapping of the areas follows an ascending linear trend across the diagram. This is a particular characteristic of the modular organization with a centralized hierarchy of the cortico-cortical network of the cat. Dividing Figure 3B into equivalent regions along the ascending trend, highlighted by shaded regions, we derive a classification of the 53 cortical areas into three categories. Cortical areas at the lower-left region of the diagram are sparsely connected and preferentially linked with areas of the same modality $\left(P_{i}^{\prime}<0.33\right)$. Hence, we name them unimodal areas. Areas falling into the upperright region of the diagram correspond to the hubs of the network; they connect to $40-60 \%$ of the areas. All of them are nearly kinless $\left(P_{i}^{\prime}>0.66\right)$ spanning their links over the four modalities: visual, auditory, somatosensory-motor, and frontolimbic. We name them supramodal hubs. In the intermediate region of the diagram we find areas which are well-connected within their modality but also make a considerable number of external connections to other modalities. These are classified as multimodal areas.

This classification, as summarized in Figure 3C, is consistent with anatomical and physiological observations. Within the unimodal group we find areas known to perform basic sensory processing: the primary and secondary visual cortices (areas 17 and 18), primary auditory cortex (area $A I$ ) and primary somatosensory cortex (areas 1,2, and $3 b$ ). Specialized areas typically exhibit characteristic internal organization. The areas 17, 18, 19, 20a, 20b, and PS are known to be retinotopically organized; the primary auditory cortex $A I$ as well as $A A F, P$, and $V P$ have tonotopic maps; the somatosensory areas 1,2 , and $3 b$ contain somatotopic maps of cells responsive to cutaneous stimulation and SIV has an orderly topographic representation of the body surface (Scannell et al., 1995). Most of these areas have been classified as unimodal and a few of them lie in the intermediate multimodal category. On the other hand, all cortical hubs forming the top hierarchical module of the network, the rich-club, lie at the group of supramodal hubs. The functional role of multimodal areas is rather unclear; they are probably involved in the modulation of neuronal activities of other modalities.

\section{SUMMARY AND DISCUSSION}

In this paper we have described fundamental properties of the architecture of cortico-cortical networks: (i) cortical areas are at very short processing paths of each other (small-world property), (ii) there are many processing paths between two cortical areas, (iii) they are organized into few modules, and (iv) they contain few highly connected areas, hubs, which (v) form a rich-club at the top of the network hierarchy. Additionally, we have seen that cortical areas can be classified into three groups, according to their multisensory connectivity.

The first two properties strongly indicate that the cortex is a highly interactive system in terms of information processing. Although regions exist which are specialized in a particular function, they do not operate independently. They work under the constant influence of each other. The modular and hierarchical architecture of the cortical networks represent the physical substrate that permits the brain to simultaneously process information of different modalities (parallel processing) and to integrate that information toward the generation of a coherent, global representation of the reality. In the light of this organization, it could be envisioned that multisensory integration emerges from the collaborative function of the cortical hubs. While early sensory cortical regions perform specialized processing of the sensory input, the hubs of the network may work together to combine the multisensory information. A relevant difference is that the cortical hubs form a network module which is geographically delocalized at the cortical surface (Figure 2).

\subsection{FROM WORMS TO HUMANS}

In Section 3 we introduced the neural connectivity of the C. elegans and the human tractography, but we barely explained their network architecture. The results discussed in Section 4, although focused on the cortical network of the cat, are largely reproduced in other connectivity datasets.

The neural network of the C. elegans exhibits several topological features described above for cortical networks. Its neurons are arranged into modules, containing neural circuits which play a vital role in performing different functions: chemosensation, thermotaxis, mechanosensation, feeding, etc. (Arenas et al., 2008; Pan et al. 2010). Some of the modules also reflect a trivial spatial proximity of the neurons in the body. The network has a scale-free-like degree distribution and it contains a few hubs (Varshney et al., 2011). Out of its 300 neurons in the worm, only 4-6 neurons could be considered hubs. Our preliminary 
Functional connectivity

Neuroimaging and electrophysiological experiments measure the temporal evolution of brain activity. Functional connectivity refers to the statistical interdependence (mutual information) between time-series of different regions, thus evaluating the pair-wise degree of co-activation. observations reveal that there is a significant richclub structure in this network.

Two approaches have been used during the recent years to study the large-scale connectivity of the human brain (Bullmore and Sporns, 2009; Bressler and Menon, 2010). On the one hand, tractography studies permit to acquire an approximate draft of the cortico-cortical connectivity. Such studies have found both a either modular arrangement of the cortical areas and a group of cortical hubs which are distributed over the cortex (Hagmann et al., 2008). On the other hand, functional connectivity extracted from both electroencephalography (EEG) and functional magnetic resonance imaging (fMRI), have shown a modular and hierarchical organization of the dynamics in the working brain (Meunier et al., 2010), what reflects features of the underlying anatomical connectivity.

We can conclude that there is enough evidence to assert that, from worms to humans, the architecture of the nervous systems of animals share fundamental principles of organization. These respond to the ubiquitous functional necessities of the organisms to (i) simultaneously deal with sensory information of different character (or modality), what arises from the limitation of sensory neurons to encode information of only one type of modality, and (ii) the necessity of the organism to combine (integrate) those different information.

\subsection{LOCALIZED VS. NETWORKED PROCESSING?}

The results presented here are summarized are to be embodied into a broader and longstanding debate. On the one hand, both physiological experiments and the study of patients with localized brain lesions have long evidenced that brain and cortical regions specialize in particular functions. On the other hand, during the last two decades, micro-electrode recordings at multiple sites and neuroimaging studies, have shown that distant regions of the brain undergo transient states of correlated activity. Based in these observations, a networked perspective has started to dominate (Knight, 2009) in which brain activity is regarded as functional networks which rapidly emerge and dissolve, governed by coordination dynamics according to the sensory stimulation and the ongoing activity (Bressler and Kelso, 2001).

Several models have been proposed that high-level functions are represented by distributed, interactive, and overlapping networks of neurons, which transcend any of the traditional subdivisions of the cortex by structural (cytoarchitecture) or functional criteria (Damasio,
1989; Fuster, 2003; Tononi, 2004; Baars, 2005; Shanahan, 2007).

Now, studying the anatomical connectivity of cortical and neural networks we find that, indeed, the nervous system is organized such that both approaches coexist. While different parts of the system specialize in performing particular functions, brain function is to be understood as emerging from the collective working of its constituents without a single coordinating center. The modular organization of the neural connectivity supports the specialization of different parts, and the highly interconnected hubs are responsible for the integration and/or coordination.

\subsection{LIMITATIONS AND OUTLOOK}

Currently available cortico-cortical network data comprise of interconnection between cortical areas in only one cerebral hemisphere. Because of the known interhemisphere differences in many mammals, particularly in humans, it will be very valuable in the future to acquire the connectivity within and between both hemispheres in animal and human models. Another relevant problem is that, modification of the parcelation used to describe cortical areas influences the connectivity of the areas, and hence, the network topology. It is therefore important to establish consistent partitions for each animal model.

It is of high relevance that future efforts are directed into achieving a map of the connectivity of the whole brain for a few mammal species, particularly at the level of the major communication routes. These maps should include the connectivity between all the main parts of the brain (hippocampus, thalamus, cerebellum, amygdala, cerebral cortex, etc.), and the functional subdivisions which can be identified within them, e.g., cortical areas. Such maps of brain connectivity will help to understand how the different behavioral responses (emotions, cognition, movement control, memory formation, etc.) coexist and interact both at the brain and at the mental levels.

\section{ACKNOWLEDGMENTS}

The authors thank Javier Borge-Holtoefer, Alex Arenas, and Sergio Gómez for valuable discussions about the re-parametrization of the roles. G. Zamora-López and J. Kurths are supported by the federal ministry of education and research, Germany (BCCN2, grant No. 01GQ1001A) and the Deutsche Forschungsgemeinschaft, research group FOR 868 (contract No. KU 837/23-2). C. S. Zhou is supported by the Hong Kong Baptist University and the Research Grants Council of Hong Kong (GRF 202710). 


\section{REFERENCES}

Achard, S., Salvador, R., Whitcher, B., Suckling, J., and Bullmore, E. (2006). A resilient, low-frequency, small-world human brain functional network with highly connected association cortical hubs. J. Neurosci. 26, 63-72.

Allman, B. L., Bittencourt-Navarrete, R.E., Keniston, L. P., Medina, A. E., Wang, M. Y., and Meredith, M. A. (2008). Do cross-modal projections always result in multisensory integration? Cereb. Cortex 18, 2066-2076.

Arenas, A., Borge-Holtoefer, J., Gómez, S., and Zamora-López, G. (2010). Optimal map of the modular structure of complex networks. New J. Phys. 12, 053009.

Arenas, A., Fernández, A., and Gómez, S. (2008). A complex network approach to the determination of functional groups in the neural system of Caenorhabditis elegans. Lect. Notes Comput. Sci. 515, 9-18.

Baars, B. J. (2005). Global workspace theory of consciousness: toward a cognitive neuroscience of human experience. Prog. Brain Res. 150, 45-53.

Barabási, A. L., and Albert, R. (1999). Emergence of scaling in random networks. Science 286, 509-512.

Basser, P. J., and Jones, D. K. (2002). Diffusion-tensor MRI: theory, experimental design and data analysis - a technical review. NMR Biomed. 15, 456-467.

Bear, M. F., Connors, B. W., and Paradiso, M. A. (2006). Neuroscience: Exploring the Brain. Baltimore, MD: Lippincott Williams and Wilkins.

Boccaletti, S., Latora, V., Moreno, Y., Chavez, M., and Hwang, D.-U. (2006). Complex networks: structure and dynamics. Phys. Rep. 424, 175-308.

Bressler, S. L. (1995). Large-scale cortical networks and cognition. Brain Res. Rev. 20, 288-304.

Bressler, S. L., and Kelso, J. A. S. (2001). Cortical coordination dynamics and cognition. Trends Cogn. Sci. (Regul. Ed. ) 5, 26-36.

Bressler, S. L., and Menon, V. (2010). Large-scale brain networks in cognition: emerging methods and principles. Trends Cogn. Sci. (Regul. Ed.) 14, 277-290.

Bullmore, E., and Sporns, O. (2009). Complex brain networks: graph theoretical analysis of structural and functional systems. Nat. Rev. Neurosci. $10,1$.

Burns, G. A. P. C., and Young, M.P.(2000). Analysis of the connectional organization of neural systems associated with the hippocampus in rats. Philos. Trans. R. Soc. Lond. B Biol. Sci. 355, 55-70.

Chung, H.-W., Chou, M.-C., and Chen, C.-Y. (2011). Principles and limitation of computational algorithms in clinical diffusion tensor MR tractography. AJNR Am. J. Neuroradiol. 32, 2-13.

Damasio, A. R. (1989). Time-locked multiregional retroactivation: a systems-level proposal for the neuronal substrates of recall and recognition. Cognition 33, 25-62.

Driver, J., and Noesselt, T. (2008). Multisensory interplay reveals crossmodal influences of "sensory-specific" brain regions, neural responses and judgments. Neuron 57, 11-23.

Driver, J., and Spence, C. (2000) Multisensory perception: beyond modularity and convergence. Curr. Biol. 10, R731-R735.

Durbin, R. M. (1987). Studies on the Development and Organisation of the Nervous System of Caenorhabditis elegans. Ph.D. thesis, King's College, Oxford.

Dyrby, T. B., Søgaard, L. V., Parker, G. J., Alexander, D. C., Lind, N. M., Baaré, W. F. C., Hay-Schmidt, A., Eriksen, N., Pakkenberg, B., Paulson, O. B. and Jelsing, J. (2007). Validation of in vitro probabilistic tractography. Neuroimage 37, 1267-1277.

Engel, A. K., and Singer, W. (2001) Temporal binding and neural correlates of sensory awareness. Trends Cogn. Sci. (Regul. Ed.) 5, 16-25.

Fahle, M. (1993). Figure-ground discrimination from temporal information. Proc. R. Soc. Lond. B Biol. Sci. 254, 199-203.

Fuster, J. M. (2003). Cortex and Mind: Unifying Cognition. New York: Oxford University Press.

Gigandet, X., Hagmann, P., Kurant, M. Cammoun, L., Meuli, R., and Thiran, J.-P. (2008). Estimating the confidence level of white matter connections obtained with MRI tractography. PLoS ONE 3, e4006. doi: 10.1371/journal. pone.0004006

Goldman-Rakic, P.S. (1988). Topography of cognition: parallel distributed networks in primate association cortex. Annu. Rev. Neurosci. 11, 137-156.

Guimerà, R., and Amaral, L. A. N. (2005) Cartography of complex networks: modules and universal roles. J. Stat. Mech. 5, P02001.

Hagmann, P., Cammoun, L., Gigandet, X., Meuli, R., Honey, C. J., Wedeen, V. J., and Sporns, O. (2008). Mapping the structural core of human cerebral cortex. PLoS Biol. 6, e159. doi: 10.1371/ journal.pbio.0060159

Hilgetag, C. C., Burns, G. A. P. C., O'neill, M. A., Scannell, J. W., and Young, M. P. (2000). Anatomical connectivity defines the organization of clusters of cortical areas in the macaque monkey and the cat. Philos. Trans. R. Soc. Lond. B Biol. Sci. 355, 91-110.
Hilgetag, C.-C., and Grant, S. (2010) Cytoarchitectural differences are a key determinant of laminar projection origins in the visual cortex. Neuroimage 51, 1006-1017.

Hilgetag, C. C., and Kaiser, M. (2004). Clustered organisation of cortical connectivity. Neuroinformatics 2 353-360.

Junker, B. H., and Schreiber, F. (ed.) (2008). Analysis of Biological Networks. Hoboken, NJ: Wiley-Interscience.

Kaiser, M. (2007). Brain architecture: a design for natural computation. Philos. Transact. A Math. Phys. Eng. Sci. 365, 3033-3045.

Kaiser, M., Martin, R., Andras, P., and Young, M. P. (2007). Simulation of robustness against lesions of cortical networks. Eur. J. Neurosci. 25 3185-3192.

Kandel, E. R., Schwartz, J.H., and Jessell, T. M. (2000). Principles of Neural Science. New York, NY: McGraw-Hill.

Knight, R. T. (2009). Neural networks debunk phrenology. Science 316, 1578

Meredith, M. A., Keniston, L. R., Dehner L. R., and Clemo, H. R. (2006) Crossmodal projections from somatosensory area SIV to the auditory field of the anterior ectosylvian sulcus (FAES) in cat: further evidence for subthreshold forms of multisensory processing. Exp. Brain Res. 172, 472-484.

Meunier, D., Lammbiotte, R., and Bullmore, E. T. (2010). Modular and hierarchically modular organization of brain networks. Front. Neurosci. 4:200. doi: 10.3389/fnins.2010.00200

Newman, M. E. J. (2003). The structure and function of complex networks. SIAM Rev. 45, 167-256.

Pan, R. K., Chatterjee, N., and Sinha, S (2010). Mesoscopic organization reveal the constraints governing Caenorhabditis elegans nervous system. PLoS ONE5, e9240. doi: 10.1371/ journal.pone.0009240

Robertson, L. C. (2003). Binding, spatia attention and perceptual awareness. Nat. Rev. 4, 93-102.

Scannell, J. W., Blakemore, C., and Young, M. P. (1995). Analysis of connectivity in the cat cerebral cortex. J. Neurosci. $15,1463-1483$.

Scannell, J.W., Burns, G. A. P.C., Hilgetag C. C., O'Neil, M. A., and Young, M. P. (1999). The connectional organization of the cortico-thalamic system of the cat. Cereb. Cortex 9, 277-299.

Scannell, J. W., and Young, M. P. (1993). The connectional organization of neural systems in the cat cerebral cortex. Curr. Biol. 3, 191-200.

Shanahan, M. (2007). A spiking neuron model of cortical broadcast and competition. Conscious. Cogn. 17, 288-303.
Singer, W., and Gray, C. M. (1995). Visual feature integration and the temporal correlation hypothesis. Annu. Rev. Neurosci. 18, 555-586.

Sporns, O., Chialvo, D. R., Kaiser, M., and Hilgetag, C.-C. (2004). Organization, development and function of complex brain networks. Trends Cogn. Sci. (Regul. Ed.) 8, 418-425.

Sporns, O., Honey, C. J., and Kötter, R. (2007). Identification and classification of hubs in brain networks. PLoS ONE 10, e1049. doi: 10.1371/journal. pone.0001049

Sporns, O., Tononi, G., and Kötter, R. (2005). The human connectome: a structural description of the human brain. PLoS Comput. Biol. 1, e42. doi: 10.1371/journal.pcbi.0010042

Sporns, O., and Tononi, G. M. (2001). Classes of network connectivity and dynamics. Complexity 7, 28-38.

Stephan, K. E., Kamper, L., Bozkurt, A. Burns, G. A., Young, M. P., and Kötter, R. (2001). Advance database methodology for the collation of connectivity data on the macaque brain. Philos. Trans. R. Soc. Lond. B Biol. Sci. 356, 1159-1186.

Tass, P., Rosenblum, M., Weule, J., Kurths, J., Pikovsky, A. S., Volkmann, J., Schnitzler, A., and Freund, H.-J. (1998). Detection of n:m phase locking from noisy data: application to magnetoencephalography. Phys. Rev. Lett. 81, 3291-3294.

Tononi, G. (2004). An information integration theory of consciousness. BMC Neurosci. 5, 42. doi: 10.1186/1471-2202-5-42

Tononi, G., Sporns, O., and Edelman, G M. (1994). A measure for brain complexity: relating functional segregation and integration in the nervous system. Proc. Natl. Acad. Sci. U.S.A 91, 5033-5037.

Travers, J., and Milgram, S. (1969). An experimental study of the small world problem. Sociometry 32, 425-443.

Uhlhaas, P. J., Roux, F., Rodriguez, E. Rotarska-Jagiela, A., and Singer, W. (2010). Neural synchrony and the development of cortical networks. Trends Cogn. Sci. (Regul.Ed.) 14,72-80.

Varela, F., Lachaux, J.-P., Rodriguez, E., and Martinerie, J. (2001). The brainweb: phase synchronization and largescale integration. Nat. Rev. Neurosci. 2, 229-239.

Varshney, L. A., Chen, B. L., Paniagua, E., Hall, D. H., and Chklovskii, D. B. (2011). Structural properties of the Caenorhabditis elegans neuronal network. PLoS Comput. Biol. 7, e1001066. doi: 10.1371/journal. pcbi. 1001066

Wallace, M. T. (2004). The development of multisensory processes. Cogn. Process. 5, 69-83. 
Wasserman, S., and Faust, K. (1994). Social Network Analysis. Cambridge: Cambridge University Press.

Watts, D. J., and Strogatz, S. H. (1998). Collective dynamics of "small-world" networks. Nature 393, 440.

White, J. G., Southgate, E., Thomson, J. N., and Brenner, S. (1986). The structure of the nervous system of the nematode Caenorhabditis elegans. Philos. Trans. R. Soc. Lond. B Biol. Sci. 314, 1-340.

Young, M. P. (1992). Objective analysis of the topological organization of the primate cortical visual system. Nature 358, 152-155.

Young, M. P. (1993). The organization of neural systems in the primate cerebral cortex. Proc. R. Soc, Lond. B Biol. Sci. 252, 13-18.

Zamora-López, G. (2009). Linking Structure and Function of Complex Cortical Networks. Ph.D. thesis, University of Potsdam, Potsdam.

Zamora-López, G., Zhou, C. S., and Kurths, J. (2009). Graph analysis of cortical networks reveals complex anatomical communication substrate. Chaos 19, 015117.

Zamora-López, G., Zhou, C. S., and Kurths, J. (2010). Cortical hubs form a module for multisensory integration on top of the hierarchy of cortical networks. Front. Neuroinform. 4:1. doi 10.3389/neuro.11.001.2010
Zemanová, L., Zhou, C. S., and Kurths, J. (2006). Structural and functional clusters of complex brain networks. Physica D 224, 202-212.

Zhou, C.S.,Zemanová,L., Zamora-López, G., Hilgetag, C. C., and Kurths, J. (2006). Hierarchical organization unveiled by functional connectivity in complex brain networks. Phys. Rev. Lett. 97, 238103

Conflict of Interest Statement: The authors declare that the research was conducted in the absence of any commercial or financial relationships that could be construed as a potential conflict of interest.
Received: 22 November 2010; accepted: 09 June 2011; published online: 21 June 2011.

Citation: Zamora-López G, Zhou C and Kurths J (2011) Exploring brain function from anatomical connectivity. Front. Neurosci. 5:83. doi: 10.3389/ fnins.2011.00083

Copyright (c) 2011 Zamora-López, Zhou and Kurths. This is an open-access article subject to a non-exclusive license between the authors and Frontiers Media $S A$, which permits use, distribution and reproduction in other forums, provided the original authors and source are credited and other Frontiers conditions are complied with. 